\title{
Paradoxical coronary artery embolism causing acute myocardial infarction in a young woman with factor $V$ Leiden thrombophillia
}

\author{
${ }^{1} \mathrm{AP}$ Croft, ${ }^{2} \mathrm{~N}$ Khan, ${ }^{2} \mathrm{MV}$ Chittari, ${ }^{3} \mathrm{C}$ Varma \\ ${ }^{1}$ Academic Clinical Fellow in Rheumatology; ${ }^{2}$ Specialist Registrar in Cardiology; ${ }^{3}$ Consultant Cardiologist, University of Birmingham \\ Department of Medicine and Department of Cardiology, City Hospital, Birmingham, UK
}

\begin{abstract}
Paradoxical coronary artery embolism is a rare but under-diagnosed cause of acute myocardial infarction (AMI) and requires a high level of clinical suspicion to make an early diagnosis. We describe the case of a young woman who presented with a severe cough and chest pain who was subsequently found to have a paradoxical embolus in the right coronary artery. Echocardiography showed a patent foramen ovale (PFO) and an atrial septal aneurysm (ASA). The patient was found to be a heterozygous carrier of the factor $V$ Leiden mutation that increases the risk for venous-thromboembolism. The association between a PFO and an ASA is a risk factor for systemic embolisation. This is the first reported case of paradoxical coronary artery embolus causing AMI in a non-pregnant patient with factor Leiden thrombophilia. Identification of this clinical phenotype is vital as the risk of future embolic events can be reduced by anticoagulation and closure of anatomical cardiac defects.
\end{abstract}

KEYWORDS Myocardial infarction, pulmonary embolus, patent foramen ovale, coronary artery embolus, paradoxical embolus, cough

DECLARATION OF INTERESTS No conflicts of interest declared.
Correspondence to AP Croft, Centre for Translational Inflammation Research, University of Birmingham Research Laboratories, Queen Elizabeth Hospital, Mindelsohn Way, Edgbaston, Birmingham BI5 2WB, UK e-mail adampcroft@doctors.org.uk

\section{CASE HISTORY}

A 44-year old non-smoking patient (Caucasian female; 72 kilograms [kg]; body mass index [BMI] 28) presented to hospital with a three-week history of increasing breathlessness and severe dry cough. She was treated with three consecutive courses of oral antibiotics (amoxicillin 500 milligrams [mg] three times daily for five days, doxycycline $100 \mathrm{mg}$ once daily and erythromycin $500 \mathrm{mg}$ four times daily for two days which were stopped due to gastrointestinal side-effects) and a fiveday course of oral prednisolone with minimal symptomatic improvement. On closer questioning the patient described severe central chest tightness radiating to the back. She had no haemoptysis or known risk factors for venous thromboembolism. On admission she was taking no regular medications or oral contraceptive pill. Her only past medical history of note was a previous episode of acute onset vertigo with ataxia that was diagnosed as a probable transient ischaemic attack (TIA) in 1995. She had also been investigated seven years previously for persistent breathlessness and had normal computed tomography (CT) pulmonary angiogram and cardiac catheterisation studies.

Her general and cardiac examination was unremarkable. Blood pressure was 109/63 millimetres of mercury (mm $\mathrm{Hg}$ ) and radial pulse was regular at a rate of 92 beats per minute with a respiratory rate of 20 breaths per minute and oxygen saturations on pulse oximetry of $95 \%$ on room air. She was afebrile on admission. Initial laboratory investigations of full blood count and biochemistry were within normal limits, apart from mildly elevated C-reactive protein levels at 30 milligrams per litre $(\mathrm{mg} / \mathrm{L})$. Arterial blood gas analysis on air was unremarkable, with no evidence of hypoxia. Serum cardiac troponin $\mathrm{T}$ levels were markedly raised at 2.47 micrograms per litre $(\mu \mathrm{g} / \mathrm{L})$ (normal range $<0.0 \mathrm{l} \mu \mathrm{g} / \mathrm{L}$ ). The plain chest radiograph was normal. The 12-lead electrocardiogram showed sinus bradycardia (49 beats per minute) with incomplete right bundle-branch block, $\mathrm{Q}$ wave in lead III and T-wave inversion in inferior and lateral leads. Transthoracic echocardiography showed mild right heart dilatation (Figure la) and moderate tricuspid regurgitation with mildly elevated peak pulmonary artery pressures of $40 \mathrm{~mm} \mathrm{Hg}$. In view of the possibility of pulmonary embolism a CT pulmonary angiography was performed and confirmed scattered pulmonary emboli in the segmental arteries of both lower lobes and the right middle lobe. However as a result of the significantly raised serum cardiac enzyme levels and possible ischaemic changes seen on the electrocardiogram (ECG), a diagnostic coronary angiography was then performed. This revealed a single discrete embolus in the distal right coronary artery (RCA) (Figure Ib). The coronary arteries were otherwise normal. A diagnosis of non-ST segment elevation myocardial infarction (NSTEMI) was made. 


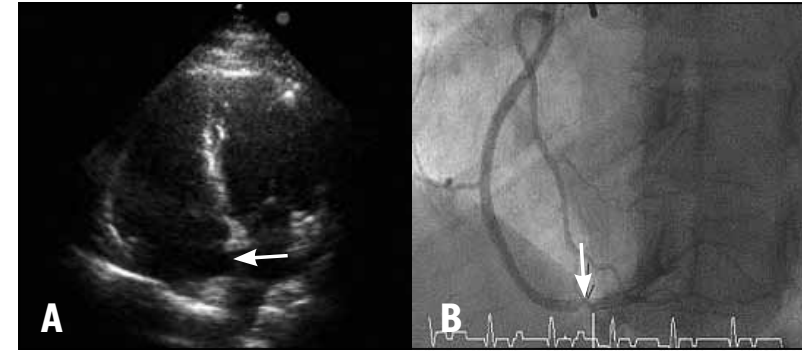

FIGURE IA Apical four-chamber view on transthoracic echocardiogram showing right heart dilatation and the presence of an interatrial septal aneurysm (arrow). FIGURE IB Coronary angiogram, left anterior oblique (LAO) cranial view demonstrating a space-occupying lesion in the distal right coronary artery, consistent with an embolus (arrow).

In order to determine the mechanism for her coronary embolus, a bubble contrast echocardiogram using the Valsalva manoeuvre was performed. This demonstrated crossover of agitated saline contrast bubbles across the interatrial septum consistent with an interatrial communication. Transoesophageal echocardiography (TOE) confirmed an atrial septal aneurysm (ASA) with a patent foramen ovale (PFO). The diagnosis was NSTEMI secondary to a paradoxical coronary artery embolus. The mechanism of embolisation was thought to be due to raised right heart pressure secondary to pulmonary embolism and severe cough facilitating right to left shunting of blood. A thrombophilia screen was performed as there were no identifiable risk factors for venous thromboembolism and her past medical history suggested a previous embolic event resulting in cerebral infarction (previous TIA). The screen demonstrated the presence of activated protein-C (APC) resistance (failure to inhibit factor $\mathrm{Va}$ and prolong coagulation when added to the individual's plasma in vitro) consistent with a diagnosis of factor $V$ Leiden deficiency. Targeted mutation analysis of the F5 gene-encoding factor $V$ confirmed the patient was heterozygous for the factor $V$ Leiden allele. The patient was commenced on anticoagulation therapy with warfarin and the PFO was subsequently closed with a percutaneous device.

\section{DISCUSSION}

Acute myocardial infarction (AMI) secondary to a paradoxical coronary artery embolus in a patient with factor $V$ Leiden thrombophilia has only been reported in the medical literature in the context of pregnancy.' This is the first reported case in a non-pregnant woman.

Paradoxical coronary embolism is a rare but underdiagnosed cause of $\mathrm{AMI}^{2}$ and requires a high index of clinical suspicion to make the diagnosis early. Recognition of this clinical phenotype is reliant on initial clinical triage and the use of appropriate cardiac investigations and imaging modalities. Serum troponin $T$ is a widely established, sensitive biochemical marker for myocardial injury. However, elevated levels do not define the mechanical injury. ${ }^{3}$ In this case, clinical presentation was further complicated by the presence of pulmonary emboli. Pulmonary embolus can lead to increased troponin but levels in excess of $1.0 \mu \mathrm{g} / \mathrm{L}$ have rarely been reported even in the presence of right ventricular dilatation. ${ }^{4}$ Therefore in the case of our patient it was important not only to consider one unifying diagnosis but to also exclude other differential diagnoses for her raised serum troponin, including AMI. Diagnosis of myocardial infarction (MI) secondary to a paradoxical coronary embolus can be missed in patients with coexistent pulmonary embolism because of the similarity in clinical symptoms and biochemical findings. ${ }^{5}$ Early echocardiography is useful in this regard.

Paradoxical embolism occurs through a PFO or cardiac septal defect in prothrombotic states when right atrial pressure exceeds the left atrial pressure resulting in paradoxical shunting of blood through the anatomical defect and passage of a thrombus of venous origin to the left atria and ultimately the systemic circulation. This reversal in pressure gradient occurs following the Valsalva manoeuvre and with pulmonary hypertension secondary to recurrent pulmonary embolism.

The most common cause of right-to-left intracardiac shunting is a PFO. ${ }^{6}$ A PFO is a component of the fetal circulation that closes by fusion of the septum primum and septum secundum shortly after birth. However this doesn't happen in about $25-30 \%$ of individuals. ${ }^{6} \mathrm{~A}$ PFO is detected in 10-15\% of the general population by contrast echocardiography ${ }^{7}$ but autopsy studies report a $27 \%$ prevalence of probe-patent foramen ovale. ${ }^{8}$ The reported prevalence is higher in patients under the age of 55 years with cryptogenic stroke (40\% of individuals have a PFO) ${ }^{9}$ and patients with migraine with aura (52\% of individuals). ${ }^{10}$

An ASA is when part or all of the atrial septum shows aneurysmal dilatation protruding into either atria during the cardiac cycle. The frequency of ASA is higher in patients evaluated with TOE after a cerebral ischaemic event than in the general population." Since ASA is frequently associated with right-to-left interatrial shunting and often associated with large PFOs, it is reported that the mechanism of cardio-embolism is most likely paradoxical embolisation through a coexistent PFO.'2

In our patient two clinical features increased our suspicion of a paradoxical coronary embolus. First, the presence of bilateral chronic pulmonary emboli with dilated right heart chambers consistent with a degree of pulmonary hypertension and secondly, a significantly raised cardiac troponin $\mathrm{T}$ level suggesting $\mathrm{MI}$ in a young woman with no other risk factors for coronary disease. We presumed that the high right atrial pressure resulting 
from pulmonary embolism and the associated pulmonary hypertension is likely to have resulted in a right-to-left shunt through a PFO. Vigorous coughing may have further exacerbated shunting.

Congenital thrombophilia is an important risk factor to identify in patients with a PFO as the risk of paradoxical embolus is significantly increased.13 Factor $V$ Leiden deficiency confers resistance of factor $V$ to degradation by activated protein-C. This results in a prothrombotic tendency, increasing the risk of venous thromboembolism four to eight fold in heterozygous carriers of the mutation. ${ }^{14}$ In addition, the association between a PFO and an atrial septal aneurysm is a strong risk factor for systemic embolisation. There is currently no evidence that the risk of venous thromboembolism is high enough

\section{REFERENCES}

I Agostoni P, Gasparini G, Destro G. Acute myocardial infarction probably caused by paradoxical embolus in a pregnant woman. Heart 2004; 90:e I 2. http://dx.doi.org/I0.I I36/hrt.2003.026526

2. Wachsman DE, Jacobs AK. Paradoxical coronary embolism: a rare cause of acute myocardial infarction. Rev Cardiovasc Med 2003; 4:107-II.

3 Jaffe AS, Ravkilde J, Roberts $\mathrm{R}$ et al. It's time for a change to a troponin standard. Circulation 2000; 102:1216-20. http://dx.doi. org/I0.II6I/0I.CIR.I02.II.I2I6

4 Janata K, Holzer M, Laggner AN et al. Cardiac troponin T in the severity assessment of patients with pulmonary embolism: cohort study.BMJ 2003; 326:3 I 2-3.http://dx.doi.org/I0.I I36/bmj.326.7384.3 I2

5 Haghi D, Sueselbeck T, Papavassiliu T et al. Paradoxical coronary embolism causing non-ST segment elevation myocardial infarction in a case of pulmonary embolism. Z Kardiol 2004; 93:824-8. http:// dx.doi.org//0.1007/s00392-004-0130-0

6 Hara H,Virmani, R, Ladich E et al. Patent foramen ovale: current pathology, pathophysiology, and clinical status. J Am Coll Cardiol 2005; 46: I 768-76. http://dx.doi.org/10.1016/j.jacc.2005.08.038

7 Di Tullio M, Sacco RL, Venketasubramanian N et al. Comparison of diagnostic techniques for the detection of a patent foramen ovale in stroke patients. Stroke 1993; 24:1020-24. http://dx.doi. org/I0.II6I/0I.STR.24.7.1020

8 Hagen PT, Scholz DG, Edwards WD. Incidence and size of patent foramen ovale during the first 10 decades of life: an autopsy study of 965 normal hearts. Mayo Clin Proc 1984; 59:17-20. to warrant long-term anticoagulation in heterozygous carriers of the factor $V$ mutation following the first thrombotic episode or in those who are asymptomatic. Long-term oral anticoagulation is considered in those with recurrent venous thromboembolism (VTE), multiple thrombophillic disorders, or coexistent circumstantial risk factors. ${ }^{15}$ This decision must be based on an individual risk vs benefit analysis.

Paradoxical coronary artery embolism is a rare cause of MI but needs to be recognised as these patients require therapy in the form of anticoagulation and/or surgical closure of an anatomical defect to reduce the risk of future embolic events.

9 Lechat P, Mas JL, Lascault G et al. Prevalence of patent foramen ovale in patients with stroke. N Engl J Med 1988; 318:1|48-52. http://dx.doi.org/I0.1056/NEJMI98805053/8I802

10 Reisman M, Christofferson RD, Jesurum J et al. Migraine headache relief after transcatheter closure of patent foramen ovale. J Am Coll Cardiol 2005;45:493-5. http://dx.doi.org/ I0.1016/j.jacc.2004.10.055

I I Mattioli AV, Aquilina M, Oldani A et al. Atrial septal aneurysm as a cardioembolic source in adult patients with stroke and normal carotid arteries. A multicentre study. Eur Heart J 200I; 22:26I-8. http://dx.doi.org/I0.1053/euhj.2001.2293

12 d'AudiffretA, Pillai L, Dryjski M.Paradoxical emboli: the relationship between patent foramen ovale, deep vein thrombosis and ischemic stroke. Eur J Vasc Endovasc Surg 1999; 17:468-7I. http:// dx.doi.org/I0.1053/ejvs. 1999.0776

I 3 Karttunen V, Hiltunen L, Rasi V et al. FactorV leiden and prothrombin gene mutation may predispose to paradoxical embolism in subjects with patent foramen ovale. Blood Coagul Fibrinolysis 2003; 14:26I-8. http://dx.doi.org//0.1097/0I.mbc.000006/288.28953.c8

14 Calderwood CJ, Greer IA. The role of factor $V$ leiden in maternal health and the outcome of pregnancy. Curr Drug Targets 2005; 6:567-76. http://dx.doi.org/ 10.2 I74// 389450054546024

15 British Society for Haematology. Investigation and management of heritable thrombophilia. Br J Haematol 2001; I 14:512-28. http:// dx.doi.org/I0.1046/j.I365-2 I4I.200I.0298I.x 